\title{
In vivo tumor targeting and anti-tumor effects of 5-fluororacil loaded, folic acid targeted quantum dot system
}

\begin{abstract}
In this study, we modulated the anti-cancer efficacy of 5-Fluorouracil (5-FU) using a carrier system with enhanced targeting efficacy towards folate receptors (FRs) expressing malignant tissues. The 5-FU drug was loaded onto Mn-ZnS quantum dots (QDs) encapsulated with chitosan (CS) biopolymer and conjugated with folic acid (FA) based on a simple wet chemical method. The formation of 5-FU drug loaded composite was confirmed using Fourier transform infrared spectroscopy (FTIR), thermo gravimetric analysis (TGA) and differential scanning calorimetry (DSC). Furthermore, the in vivo biodistribution and tumor targeting specificity of the 5-FU@FACS-Mn:ZnS in the tumor-bearing mice was conducted based on the $\mathrm{Zn} 2+$ tissue bioaccumulation using inductively coupled plasma (ICP) spectroscopy. In addition to the characterization, the in vitro release profile of 5-FU from the conjugates investigated under diffusion controlled method demonstrated a controlled release behaviour as compared against the release behaviour of free 5-FU drug. The as-synthesized 5-FU@FACS-Mn:ZnS nanoparticle (NP) systemically induced higher level of apoptosis in breast cancer cells in vitro as compared to cells treated with free 5-FU drug following both cell cycle and annexin assays, respectively. Also, the in vivo toxicity assessment of the 5FU@FACS-Mn:ZnS NPs as compared to the control did not cause any significant increase in the activities of the liver and kidney function biomarkers, malondialdehyde (MDA) and nitric oxide (NO) levels. However, based on the FA-FRs chemistry, the 5-FU@FACS-Mn:ZnS NPs specifically accumulated in the tumor of the tumor-bearing mice and thus contributed to the smaller tumor size and less event of metastasis was observed in the lungs when compared to the tumor-bearing mice groups treated with the free 5-FU drug. In summary, the results demonstrated that the 5-FU@FACS-Mn:ZnS QDs exhibits selective anti-tumor effect in MDA-MB231 breast cancer cells in vitro and 4TI breast cancer cells in vivo, providing a blueprint for improving the 5-FU efficacy and tumor targeting specificity with limited systemic toxicity.
\end{abstract}

Keyword: 5-fluorouracil delivery; Mn:ZnS quantum dots; Folic acid; Targeted delivery; Anti-tumor; Theranostics 